\title{
Virtual computed tomographic bronchoscopy: normal bronchial anatomy in six dogs
}

\author{
Dayoung Oh, Mincheol Choi, Junghee Yoon* \\ College of Veterinary Medicine and the Research Institute for Veterinary Science, Seoul National University, \\ Seoul 08826, Korea
}

(Received: March 24, 2017; Accepted: June 5, 2017)

\begin{abstract}
The aim of this study was to examine normal bronchi in dogs by using virtual bronchoscopy (VB) and to evaluate the utility of VB in clinical practice. The bronchi of six dogs without tracheobronchial disease were visualized by VB. Airways from the tracheal bifurcation to the lobar bronchi were well visualized in all dogs. Segmental and subsegmental bronchi were also well identified, but the degree and number of those varied with dog size. The mean numbers of segmental and subsegmental bronchi identified in the six dogs were 41.83 and 50.17, respectively, whereas, the mean numbers in medium- and large-sized dogs were 55.00 and 82.67, respectively, and in small-sized dogs, the means were 28.67 and 17.67, respectively. Although there were size-dependent differences in VB visualization of the bronchi, it was possible to identify peripheral airways to the subsegmental bronchi level, which can rarely be accomplished via conventional bronchoscopy. VB is the noninvasive method that can be used to examine bronchial anatomy, and our results suggest that VB can be useful for evaluating bronchi, including segmental and subsegmental ones that cannot be examined routinely by conventional bronchoscopy. Thus, VB has potential as an alternative to conventional bronchoscopy in the examination of bronchi in dogs.
\end{abstract}

Keywords: computed tomography, dogs, normal bronchi, virtual bronchoscopy

\section{Introduction}

Diagnostic imaging including thoracic radiography, fluoroscopy and computed tomography (CT) is used to help identifying tracheobronchial diseases and conventional bronchoscopy is actually an important diagnostic technique for evaluating the accurate lesions in veterinary medicine [12]. However, this modality is invasive so complications caused by the examinations may be inevitable. Virtual bronchoscopy (VB) creates a 3-dimentional endoscopic image from $\mathrm{CT}$ data and displays the hollow lumen of bronchi as if by actual endoscopic examinations. In humans, many reports about clinical application of VB and comparison between conventional bronchoscopy and VB have been studied [4, 5]. In dogs, only two reports associated with the VB were studied that VB can be used for supportive diagnostic modality in dogs with lung lobe torsion and induced laryngotracheal stenosis [3, $10]$. The purpose of this study is to describe the normal virtual bronchoscopic appearance of canine bronchi and to assess the usefulness of VB in clinical practice in veterinary medicine.

*Corresponding author

Tel: +82-880-1265, Fax: +82-876-8662

E-mail: heeyoon@snu.ac.kr

\section{Materials and Methods}

\section{Animals}

Six patients who had thoracic CT examination for evaluating additional pulmonary lesions and metastasis related to underlying diseases and were diagnosed with no bronchial diseases were included. There were 2 castrated males, 3 spayed females, and 1 intact female. Ages were ranged from 10 months to 13 years old. The patients were a Golden Retriever with $32 \mathrm{~kg}$ (P1, a skull mass suspected to chondrosarcoma), a Welsh Corgi with $19.2 \mathrm{~kg}$ (P2, follicular cell thyroid carcinoma), a Bedlington Terrier with $10.5 \mathrm{~kg}$ (P3, salivary gland adenocarcinoma), a Schnauzer with $7.3 \mathrm{~kg}$ (P4, squamous cell carcinoma in right hindlimb phalanges), a Maltese with $3.2 \mathrm{~kg}$ (P5, chronic facial inflammation), and a Spitz with $6.6 \mathrm{~kg}$ (P6, intestinal adenocarcinoma). It was regarded as medium to large sized dogs if weighing more than $10 \mathrm{~kg}$ and as small sized dogs if weighing less than $10 \mathrm{~kg}$. According to this, P1 to P3 were classified as medium to large sized dogs and P4 to P6 as small sized dogs. All CT examinations were performed at the Veterinary Medical Teaching Hospital of Seoul National University. 


\section{CT examination and VB}

All dogs were positioned in sternal recumbency and CT images were acquired using a 64 row multidetector, CT scanner (Aquilion 65; Toshiba, Japan). CT imaging conditions were as follows: $120 \mathrm{kVp}, 150-200 \mathrm{mAs}, 0.75-1.0 \mathrm{sec} /$ rotation, $0.5-1.0 \mathrm{~mm}$ scan slice thickness without reconstruction intervals and 1.0 helical pitch. Three dimension (D) volume renderings were obtained in Digital Imaging and Communications in Medicine (DICOM) using an image processing workstation (Vitrea II ver. 4.1.0.0; Vital Images, USA).

\section{Image analysis}

The 3D images of bronchi were evaluated and the number of bronchi was counted if the bronchial orifices were clearly visualized. The bronchial anatomical structures including the tracheal bifurcation, mainstem bronchi, lobar bronchi, segmental bronchi, and if possible, subsegmental bronchi were assessed in each patient.

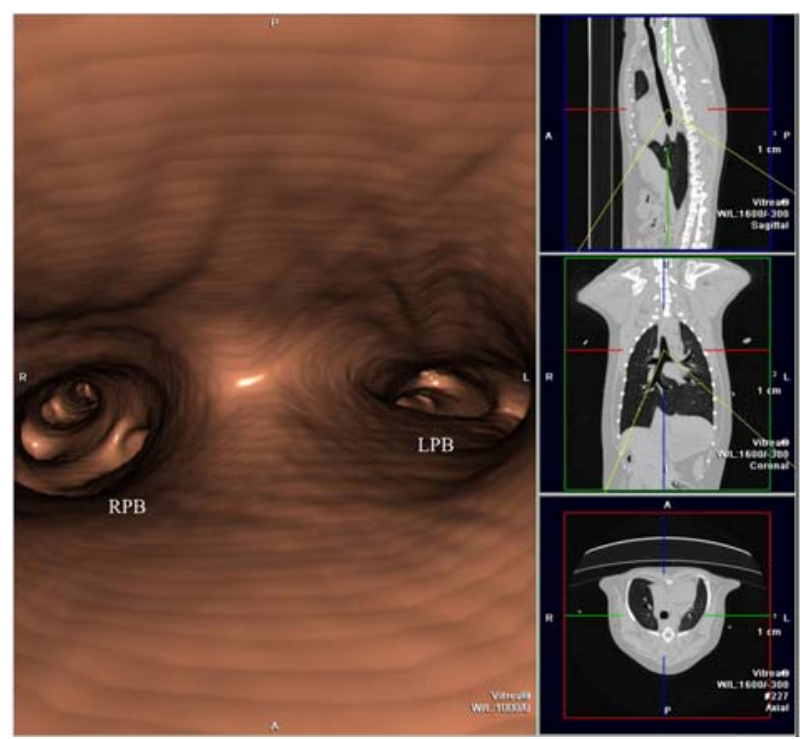

Fig. 1. The virtual bronchoscopic image of tracheal bifurcation in P1 (Golden Retriever with $32 \mathrm{~kg}$; a skull mass suspected to chondrosarcoma). The sagittal, dorsal and transverse computed chromatography (CT) 2 demension (D) images are shown together and the virtual bronchoscopic images are verified by pointing to a cursor in $2 \mathrm{D}$ view. $\mathrm{RPB}$, right principal bronchus; LPB, left principal bronchus.

\section{Results}

The tracheal bifurcation, bilateral mainstem bronchi and lobar bronchi were equally clearly visualized in all dogs (Figs. 1 and 2). Segmental and subsegmental bronchi were
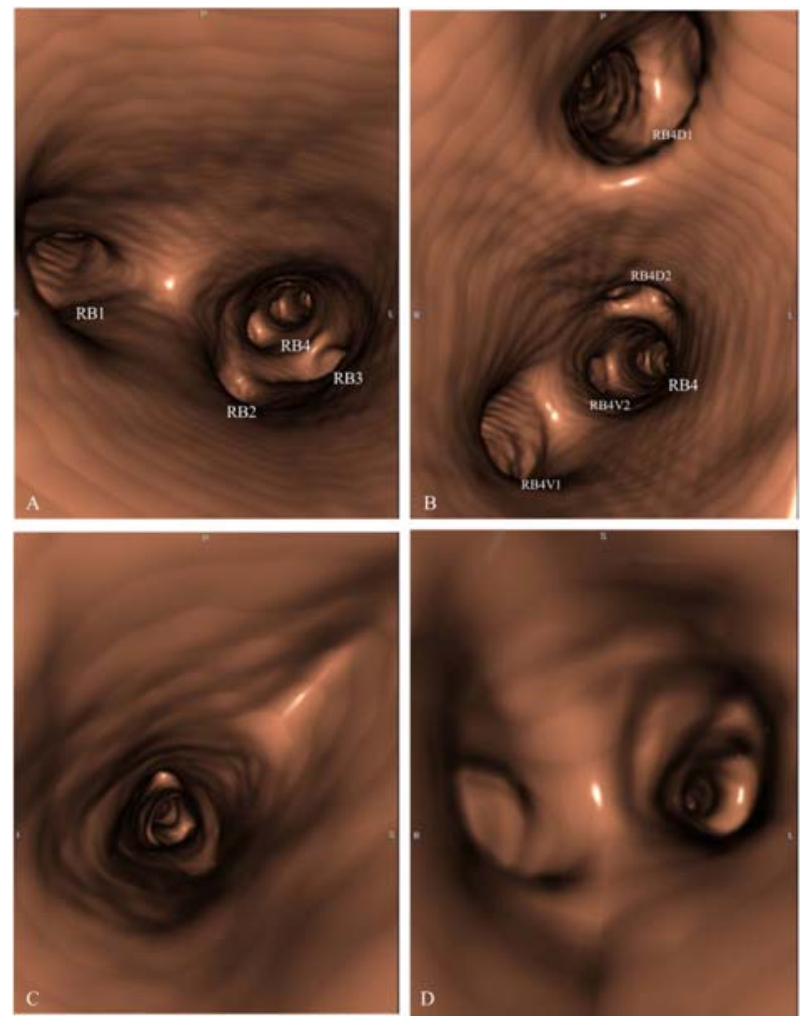

Fig 2. From the principal bronchi to subsegmental bronchi can be visualized (A) right principal bronchus, (B) right caudal lobar bronchus, (C) left caudal ventral segmental bronchus and (D) subsegmental bronchus from the left caudal dorsal segmental bronchus. RB1, right cranial lobar bronchus; RB2, right middle lobar bronchus; RB3, right accessory lobar bronchus; RB4, right caudal lobar bronchus; RB4D1, first dorsal segmental bronchus from the right caudal lung lobar bronchus; RB4D2, second dorsal segmental bronchus from the right caudal lung lobar bronchus; RB4V1, first ventral segmental bronchus from the right caudal lung lobar bronchus; RB4V2, second ventral segmental bronchus from the right caudal lung lobar bronchus.

Table 1. The number of segmental bronchi seen in each dog and mean \pm SD for each segmental bronchi $(n=6)$

\begin{tabular}{lrrrrrrr}
\hline \hline Lung lobes & P1 & P2 & P3 & P4 & P5 & P6 & Mean \pm SD \\
\hline Right cranial & 10 & 8 & 12 & 4 & 5 & 6 & $7.50 \pm 3.08$ \\
Right middle & 7 & 7 & 6 & 4 & 3 & 3 & $5.00 \pm 1.90$ \\
Right accessory & 4 & 4 & 5 & 1 & 4 & 4 & $3.67 \pm 1.37$ \\
Right caudal & 15 & 15 & 13 & 4 & 8 & 9 & $10.67 \pm 4.41$ \\
Left cranial & 5 & 5 & 8 & 3 & 5 & 4 & $5.00 \pm 1.67$ \\
Left caudal & 15 & 15 & 13 & 4 & 5 & 10 & $10.33 \pm 4.89$ \\
\hline Total & 56 & 54 & 55 & 20 & 30 & 36 & $41.83 \pm 15.32$ \\
\hline
\end{tabular}


Table 2. The number of subsegmental bronchi seen in each dog and mean \pm SD for each subsegmental bronchi $(n=6)$

\begin{tabular}{lrrrrrrr}
\hline \hline Lung lobes & P1 & P2 & P3 & P4 & P5 & P6 & Mean \pm SD \\
\hline Right cranial & 13 & 4 & 18 & 4 & 0 & 5 & $7.33 \pm 6.74$ \\
Right middle & 3 & 6 & 10 & 0 & 4 & 5 & $4.67 \pm 3.33$ \\
Right accessory & 5 & 6 & 6 & 0 & 2 & 0 & $3.17 \pm 2.86$ \\
Right caudal & 38 & 24 & 23 & 9 & 2 & 5 & $16.83 \pm 13.85$ \\
Left cranial & 2 & 5 & 17 & 0 & 2 & 3 & $4.83 \pm 6.18$ \\
Left caudal & 30 & 17 & 21 & 9 & 2 & 5 & $14.00 \pm 10.62$ \\
\hline Total & 91 & 62 & 95 & 22 & 8 & 23 & $50.17 \pm 37.75$ \\
\hline
\end{tabular}

also identified in all dogs but the degree and number of those were different in each dogs (Tables 1 and 2). The segmental and subsegmental bronchi were displayed more in medium to large sized dogs (P1-P3) than in small sized (P4-P6); the mean value of the number of identified segmental and subsegmental bronchi in medium to large sized dogs was 55.00 \pm 1.00 and $82.67 \pm 18.00$ and in small sized dogs $28.67 \pm$ 8.08 and $17.67 \pm 8.39$. In addition, it was from the right and left caudal lobar bronchi that the segmental and subsegmental bronchi were best viewed.

As breathing was held during the CT examination in all dogs, minimum motion artifacts caused by respiration were occurred. The atelectasis was induced in four dogs (P2-P4 and P6) by anesthesia and this finding did not hinder viewing bronchial structures by VB.

\section{Discussion}

The bronchi are air-conducting tubes with bands of smooth muscle and flattened overlapping cartilage rings for support. The bronchial tree is consisted of left and right principal bronchi and each bronchus is divided into lobar bronchi that are subdivided into segmental bronchi and then further into subsegmental bronchi $[1,6,11]$. The threshold value between -790 and -930 was selected to assess the bronchial structures. The focus of this study was confirming the visibility of anatomical bronchi by VB. VB could be possible to visualize up to subsegmental bronchi although individual difference was existed. The segmental and subsegmental bronchi were visualized more in large sized dogs than in small sized dogs. In humans, the visibility of the bronchial virtual images was expected lower in children than in adults because of the smaller size of the airways and the same reason can be applied to the dogs [8]. Therefore, the difference in bronchial visualization seen in medium to large and small sized dogs may result from differences in bronchial size.

With 2D CT images, the evaluation of bronchial wall and diameter such as wall thickness or bronchioarterial ratio can be performed [2,14]. As the VB images are seen together with CT 2D images, the evaluation of bronchi that are abnormally narrowed, occluded or compressed can be performed more accurately [10]. In addition, the lumen of subsegmental bronchi that are too small to evaluate in detail by $2 \mathrm{D} \mathrm{CT}$ images can be assessed by using VB.

Conventional bronchoscopy is a useful tool when evaluating canine respiratory disease and when evaluating tracheobronchial structure diseases including tracheobronchial collapse, stricture and intraluminal mass, inflammatory conditions including bronchitis and pneumonia, and traumatic injuries [9]. However, despite these indications, the examination by conventional bronchoscopy cannot be proceeded routinely because it is invasive and thereby may cause complications such as exacerbation of inflammation, hemorrhage, pneumomediastinum, pneumothorax and accumulation of secretions. In addition, most patients undergoing the procedure tend to have some degree of respiratory disease, so the conditions can be worsened after the evaluation [9]. Because VB is non-invasive technique, the risk of complication with conventional bronchoscopy can be highly reduced assuming the anesthesia is performed safely. The complications related to traumatic injuries caused by the bronchoscopic tool are never occurred with VB. In small sized dogs, the airway obstruction can be caused by conventional bronchoscope because of small size of bronchi so it has to be removed every 30 to $60 \mathrm{sec}$ for ventilation [9]. With VB, there is no concern about tool-related hypoventilation and about the time limit taken to do examination up to the smaller bronchi. In humans, the research about the utility and indication of $\mathrm{VB}$ has been conducted and the possibility of using $\mathrm{VB}$ as an alternative modality of conventional bronchoscopy was suggested $[4,8,13]$. In contrast, many other studies also concluded that VB cannot replace the conventional bronchoscopy but can assist the conventional bronchoscopy and give additional information [5, 7]. In veterinary medicine, there have been no studies about direct comparison between conventional bronchoscopy and VB in clinical practice that was not performed in this study neither. But in this study the details of anatomical structures of bronchi were visualized by $\mathrm{VB}$, which may expect VB to have usefulness in helping diagnose tracheobronchial disease and potentials of an alternative modality of the conventional bronchoscopy in patients who cannot be applied to the conventional bronchoscopy. Further study will be needed to determine the obvious utility of VB compared to the conventional bronchoscopy. VB can be used to visualize the areas beyond the tracheobronchial stenosis, envision the pre-bronchoscopy planning 
and the lesions for biopsy in humans. These indications may be applied to the veterinary medicine as well $[3,13]$.

It remains a limitation that the color of lesions cannot be differentiated from the normal mucosa and dynamic bronchial lumen during normal respiratory phase by VB. The dogs only having no tracheobronchial diseases were included in this study so the abnormal bronchi could not be identified. If the further study applying VB to the patients who have the diseases is performed, the utility of VB can be determined more clearly. Also, the purpose of this study is to confirm the possibility and efficiency of VB in evaluation of bronchial anatomy, not to compare the degree of visualizing bronchi between different breed or body size of dogs. Therefore, the increase of the diversity and the size in patients would have to be included in the future study with statistical analysis to demonstrate the efficiency of VB.

In conclusion, $\mathrm{VB}$ is noninvasive and can examine the bronchial structures to the subsegmental bronchi. It is thought that VB can be used in helping evaluate the tracheobronchial diseases and further as an alternative modality of the conventional bronchoscopy in patients who cannot be applied to the conventional bronchoscopy.

\section{References}

1. Amis TC, McKiernan BC. Systematic identification of endobronchial anatomy during bronchoscopy in the dog. Am J Vet Res 1986, 47, 2649-2657.

2. Cannon MS, Wisner ER, Johnson LR, Kass PH. Computed tomography bronchial lumen to pulmonary artery diameter ratio in dogs without clinical pulmonary disease. Vet Radiol Ultrasound 2009, 50, 622-624.

3. Eliashar R, Davros W, Gramlich T, Moffett K, Eliachar I, Esclamado R, Strome M. Evaluating laryngotracheal stenosis in a canine model with virtual bronchoscopy. Ann Otol Rhinol Laryngol 2000, 109, 906-912.

4. De Wever W, Bogaert J, Verschakelen J. Virtual bronchos- copy: accuracy and usefulness-an overview. Semin Ultrasound CT MR 2005, 26, 364-373.

5. De Wever W, Vandecaveye V, Lanciotti S, Verschakelen J. Multidetector CT-generated virtual bronchoscopy: an illustrated review of the potential clinical indications. Eur Respir J 2004, 23, 776-782.

6. Evans HE, de Lahunta A. The respiratory system. In: Miller's Anatomy of the Dog. 4th ed. pp. 350-352, Elsevier, St. Louis, 2012.

7. Fleiter T, Merkle EM, Aschoff AJ, Lang G, Stein M, Görich J, Liewald F, Rilinger N, Sokiranski R. Comparison of real-time virtual and fiberoptic bronchoscopy in patients with bronchial carcinoma: opportunities and limitations. AJR Am J Roentgenol 1997, 169, 1591-1595.

8. Konen E, Katz M, Rozenman J, Ben-Shlush A, Itzchak Y, Szeinberg A. Virtual bronchoscopy in children: early clinical experience. AJR Am J Roentgenol 1998, 171, 1699-1702.

9. Rha JY, Mahony O. Bronchoscopy in small animal medicine: indications, instrumentation, and techniques. Clin Tech Small Anim Pract 1999, 14, 207-212.

10. Schultz RM, Peters J, Zwingenberger A. Radiography, computed tomography and virtual bronchoscopy in four dogs and two cats with lung lobe torsion. J Small Anim Pract 2009, 50, 360-363.

11. Schwarz T, Johnson V. Lungs and bronchi. In: Schwarz T, Saunders J (eds.). Veterinary Computed Tomography. 1st ed. pp. 261-276, John Wiley \& Sons, Chichester, 2011.

12. Padrid PA. Laryngoscopy and tracheobronchoscopy of the dog and cat. In: Tams TR, Rawlings CA (eds.). Small Animal Endoscopy. 3rd ed. pp. 331-359, Elsevier Mosby, St. Louis, 2010.

13. Vining DJ, Liu K, Choplin RH, Haponik EF. Virtual bronchoscopy. Relationships of virtual reality endobronchial simulations to actual bronchoscopic findings. Chest 1996, 109, 549-553.

14. Won S, Lee A, Choi J, Choi M, Yoon J. Computed tomographic bronchioarterial ratio for brachycephalic dogs without pulmonary disease. J Vet Sci 2015, 16, 221-224. 